\title{
EL DESARROLLO DE LA COMPETENCIA TRANSCULTURAL DURANTE EL ESTUDIO EN EL EXTRANJERO
}

\author{
Isabel Pereira \\ Asunción Martínez-Arbelaiz
}

\section{(ब) $\Theta \Theta$}

Esta obra está bajo una licencia Creative Commons 



\title{
EL DESARROLLO DE LA COMPETENCIA TRANSCULTURAL DURANTE EL ESTUDIO EN EL EXTRANJERO
}

\author{
THE DEVELOPMENT OF TRANS-CULTURAL COMPETENCY \\ WHILE STUDYING ABROAD
}

\author{
Isabel Pereira \\ Asunción Martínez-Arbelaiz
}

\begin{abstract}
RESUMEN
Es una realidad que la universidad actual trata de proporcionar facilidades económicas y académicas de forma que los alumnos universitarios hagan parte de sus estudios en otro contexto cultural diferente al propio. Las ganancias de este esfuerzo por parte de la administración y de los propios estudiantes son a menudo difíciles de cuantificar. En el campo de la adquisición de lenguas, se suele señalar que los alumnos que estudian la lengua meta rodeados de esta, son superiores a los alumnos que la estudian solamente en el aula (Howard, 2001; 2005; Martínez-Arbelaiz y Pereira, 2008; Meara, 1994; Milton y Meara, 1995; Pérez-Vidal y Juan-Garau, 2009; Pérez-Vidal, 2014; Segalowitz y Freed, 2004). Sin embargo, contamos con pocas investigaciones sobre cómo esta experiencia repercute en el desarrollo de la competencia transcultural (Modern Language Association, 2007), uno de los objetivos de cualquier currículum de lenguas. Por medio de un análisis de grabaciones de discurso de aula, observamos tres clases de tres cursos diferentes y de pequeño tamaño. Constatamos que los alumnos estadounidenses que estudian en Madrid son observadores críticos de la comunidad que les acoge y que estas observaciones les llevan a cuestionar y refinar ideas anteriores sobre la sociedad española.

Palabras clave: estudios en el extranjero, competencia transcultural, discurso de aula, faultlines discursivos, español como segunda lengua.
\end{abstract}

\begin{abstract}
It is a reality that the university today is providing economic and academic facilities for university students to do part of their studies in another cultural context different to their own. The profits of this effort from the administration as well as from the students themselves are often difficult to quantify. In the field of language acquisition, it is often believed that students studying surrounded by the target language are better than those studying it only in the classroom (Howard, 2001; 2005; Martínez-Arbelaiz \& Pereira, 2008; Meara, 1994; Milton \& Meara, 1995; Pérez-Vidal \& Juan-Garau, 2009; Pérez-Vidal, 2014; Segalowitz \& Freed, 2004). However, there are few studies that have investigated what kind of impact this experience abroad has on the development of students' transcultural competence (Modern Language Association, 2007), one of the main goals of any language curriculum. In three filmed classes from three different small size courses we analyzed the classroom discourse. We found that U.S. students in Madrid are critical observers of the host community and that these observations led them to challenge and refine previous ideas on Spanish society.

Keywords: study abroad, transcultural competence, classroom discourse, discursive faultlines, Spanish as a second language.
\end{abstract}

Dra. Isabel Pereira. New York University Madrid. Subdirectora de Asuntos Académicos. España.

Correo electrónico: ip15@nyu.edu

Dra. Asunción Martínez-Arbelaiz. University Studies Abroad Consortium. Coordinadora de Lengua Española. España.

Correo electrónico: asuncion@usac.unr.edu

Recepción: 13- 05- 2015

Aceptación: 13- 09- 2015 


\section{Introducción}

Desde la perspectiva del Consejo Europeo (Takkula, Kangaslahti y Banks, 2008) todo aquel que participa en una experiencia internacional o se prepara para ella, tiene que adquirir nuevas habilidades para desarrollar una competencia croscultural o intercultural, o lo que también se viene denominando de una manera más global, la competencia comunicativa transcultural. Usemos el término que usemos, en palabras de los autores el presente desafío es ayudar a los ciudadanos a desarrollar estas competencias (p. 2). La Asociación de Lenguas Modernas (Modern Language Association, 2007, p. 4) proporciona la siguiente definición de competencia transcultural en lo concerniente al aula de idiomas:

\footnotetext{
...la habilidad para comprender y analizar las narrativas culturales que aparecen en todo tipo de forma expresiva- desde textos, ensayos, ficción, poesía, teatro, periodismo, humor, anuncios, retórica política y documentos legales hasta actuaciones, formas visuales y música. [“...the ability to comprehend and analyze the cultural narratives that appear in every kind of expressive form-from essays, fiction, poetry, drama, journalism, humor, advertising, political rhetoric, and legal documents to performance, visual forms, and music.']
}

En este estudio nos preguntamos cuál es la contribución de los estudios universitarios en el extranjero para desarrollar esta deseada competencia transcultural. A menudo se tiene una imagen simplificada y un tanto frívola de las vivencias de los universitarios que viajan a diferentes países con objeto de aprender la lengua y la cultura meta (Gore, 2005, p. 23). Sin embargo, los llamados "estudios en el extranjero" (inglés, study abroad), suponen una oportunidad de oro para mejorar las habilidades en una segunda lengua. En el campo de adquisición de segundas lenguas, diferentes estudios han mostrado que los alumnos que estudian la lengua meta rodeados de esta, obtienen mejores resultados que los alumnos que la estudian únicamente en el aula (Howard, 2001; 2005; Martínez-Arbelaiz y Pereira, 2008; Meara, 1994; Milton y Meara, 1995; Pérez-Vidal y Juan-Garau, 2009; Pérez-Vidal, 2014; Segalowitz y Freed, 2004).

Asimismo, cada vez contamos con más investigaciones que arrojan luz no solo sobre la adquisición de aspectos formales de la segunda lengua, sino también sobre el desarrollo de la competencia comunicativa transcultural durante el estudio en el extranjero. Entre estos estudios cabe destacar el llevado a cabo por Merino y Avello (2014), en el que se muestra que hay más alumnos con consciencia intercultural (Byram, 1997; Byram, Nichols y Stevens, 2001) después de una experiencia de estudio en el extranjero. Estas autoras analizan las redacciones de 28 alumnos que vivieron en un país de habla inglesa y observan que, tras esa experiencia, hay evidencia de consciencia intercultural, pero desafortunadamente esta no tiene un efecto duradero, puesto que en las redacciones escritas quince meses después, dicha evidencia desaparece.

Recientemente están apareciendo otros estudios de corte cualitativo que investigan la naturaleza de las experiencias de los aprendices en el extranjero, como el realizado por Goldoni (2013), a partir de entrevistas y observaciones a 44 alumnos estadounidenses en varios programas de enseñanza de español en España. La autora observa que algunos alumnos experimentan obstáculos para la integración y esperan que la comunidad de acogida sea similar a la suya de origen, continuando con una perspectiva etnocéntrica y buscando refugio en sus compañeros de idéntica procedencia. Por otra parte, también observa que algunos alumnos desarrollaron amistades con personas locales de su edad, por medio de voluntariado o aficiones comunes o con las familias que les acogían. Obviamente, los alumnos que comentaron en sus entrevistas estas experiencias de conexión con la comunidad, crearon oportunidades para practicar la 
lengua y disfrutar de aspectos de la cultura meta. Asimismo, Jackson (2008) realiza entrevistas en profundidad a cuatro alumnas de Hong-Kong antes, durante y después de estudiar inglés cinco semanas en Inglaterra. El estudio deja en evidencia los cambios emocionales que estas alumnas experimentan en relación con su identidad a consecuencia de este viaje. Finalmente, el detallado estudio de caso de Lily, una alumna aventajada británica en México a lo largo de un semestre (Bacon, 2002), nos muestra cómo la aprendiz se rebela hacia aspectos de la cultura meta que no encajan con su identidad, aunque al final de su estancia empieza a desarrollar una visión más matizada de la cultura mexicana.

Estos tres últimos estudios brevemente resumidos giran en torno al complejo proceso de integración de los alumnos en la comunidad meta. Como Doerr (2012; 2013) señala, la inmersión es una forma de aprendizaje altamente valorada en los estudios en el extranjero, relacionada con el aprendizaje experimental. Pero la misma Doerr (2013, p. 240) afirma que en lugar de "aprender sobre otra cultura", el proyecto de estudios en el extranjero debería permitir a los alumnos conectar con las personas que conocen, no como representantes de otra cultura sino como seres humanos con diferentes posicionamientos con los que puede que compartan ideas, sensibilidades u objetos materiales. Partiendo de la idea de Doerr, asumimos de que es a través de relaciones significativas con otras personas como se consigue esa tercera cultura o tercer espacio (Kramsch, 2009) necesario para el desarrollo de la competencia intercultural. En concreto, nos planteamos si la combinación de la intervención pedagógica y la experiencia fuera del aula que los programas de estudio en el extranjero ofrecen son suficientes para desencadenar el desarrollo de la competencia transcultural. En el siguiente apartado detallamos qué entendemos por este tipo de competencia y qué tipo de actividades pueden propiciarla en el aula.

\section{La competencia transcultural}

En el año 2007 la Asociación de Lenguas Modernas (Modern Language Association) elaboró un documento en el que se detallaron los objetivos de los estudios de lengua en las universidades estadounidenses. Podemos resumir dichos objetivos en un resultado de aprendizaje específico: el lograr que los alumnos se conviertan en hablantes educados con una profunda competencia transcultural. Se supone que los estudios de idiomas en la universidad tienen que lograr que los alumnos reflexionen sobre el mundo y sobre ellos mismos a través de la lente de otra lengua y cultura. Asimismo, deben aprender a comprender a los hablantes de la lengua meta como miembros de sociedades extranjeras y entenderse como estadounidenses, es decir, como miembros de una sociedad que a su vez es extranjera a otras.

El objetivo de este tipo de aprendizaje es considerar formas alternativas de ver, sentir y comprender las cosas y es ahí donde los cursos de contenido tienen una función primordial dentro del curriculum. "Un currículum debería consistir en una serie de cursos complementarios o vinculados que incorporen de forma holística la reflexión del contenido croscultural en cada nivel". ["A curriculum should consist of a series of complementary or linked courses that holistically incorporate content and crosscultural reflection at every level."] (p. 5), según la Modern Language Association (2007). En este sentido, cursos de historia, geografía, cultura, literatura de la comunidad o comunidades donde se habla la lengua meta son fundamentales para que los alumnos adquieran la capacidad de comprender e interpretar no solo la radio, la televisión o cualquier impreso sino también a los hablantes de dicha lengua. 
Sin embargo, investigaciones recientes han señalado que la discusión sobre temas culturales en clase no siempre lleva al desarrollo de la transculturalidad. Menard-Warwick (2009) estudia el discurso de clase con objeto de determinar si existen oportunidades de desarrollar la competencia transcultural en dichas clases. En concreto, analiza las representaciones culturales que los alumnos de inglés como segunda lengua (SL), inmigrantes en los Estados Unidos, y los alumnos de inglés como lengua extranjera (FL) en Chile se forman de las culturas meta. Para este análisis, Menard-Warwick (2009) recurre a faultlines discursivos o discursive faultlines (Kramsch, 1993), los cuales se definen como "áreas de diferencia cultural o de posibles malentendidos que se manifiestan en la conversación en el aula" ["areas of cultural difference or misunderstanding that become manifest in classroom talk"] (pp. 30-31). Esta autora encuentra que los faultlines discursivos son evidentes en el discurso de clase cuando se co-construye una representación de la vida de los Estados Unidos y de Chile, puesto que los alumnos entran en conflicto entre ellos o con el profesor. Asimismo, este estudio documenta diferencias entre los dos contextos de aprendizaje del inglés, puesto que los inmigrantes en California pudieron comparar las imágenes que los medios de comunicación proyectan de los estadounidenses con sus propias experiencias. Sin embargo, los alumnos en Chile tuvieron pocas oportunidades de establecer estos contrastes (p. 42). Menard-Warwick concluye que en las clases observadas, los alumnos y los profesores "hallan muchas formas de cuestionar y reconstruir las representaciones culturales en el transcurso del diálogo entre ellos, los textos del curso y los medios de comunicación" ["find numerous ways to question and reconstruct cultural representations as they enter into dialogue with each other, with course texts, and with the media"] (p. 44).

Sin embargo, la manera de llevarlo a cabo parece diferir entre clases de FL y SL: mientras el grupo chileno se centraba en aspectos relacionados con el cambio cultural del propio país (profesor y alumnos eran chilenos), en el grupo de California se destacaron más comparaciones entre las diferentes culturas de los alumnos (experiencias transnacionales en términos de la autora), y su comparación con EE.UU.

Menard-Warwick (2008) destaca la importancia de las experiencias transnacionales de los profesores. Estas experiencias dan pie a intercambios de percepciones culturales en los que no siempre los del mismo país estaban de acuerdo, no siempre sus opiniones eran diferentes a las estadounidenses o entre las diferentes nacionalidades presentes en la clase. Concluye, por tanto, que los profesionales de la enseñanza de inglés deben desarrollar una conciencia metacognitiva sobre los recursos culturales que los alumnos y profesores pueden compartir en el aula. Es decir, como parte de su preparación para ser profesores, no solo deben compartir sus trayectorias culturales con los alumnos, sino también recurrir a textos de memorias multiculturales o películas, para facilitar la exploración y discusión sobre los mismos desde las diferentes perspectivas del alumnado (pp. 635-637).

Como señalan Dytynyshyn y Collins (2012), parece que la clase de SL es un contexto rico para el desarrollo intercultural debido al contacto entre estudiantes de diversas culturas. Por eso, decidieron investigar una clase de inglés como SL en una situación cultural más compleja que la de la mayoría de los contextos de FL o SL: Montreal, una ciudad multiétnica de habla francesa en un país de habla inglesa. Estos autores analizaron la enseñanza de la competencia cultural e intercultural en una clase multiétnica formada por 19 alumnos de ESL en Montreal, para lo cual grabaron 38 horas de este curso. La profesora era canadiense de habla inglesa. Dytynyshyn y Collins se enfocaron en comprobar si la cultura canadiense estaba representada en la clase de ESL, en la perspectiva de la profesora sobre la enseñanza 
de la cultura, y si promovía el desarrollo de la interculturalidad. Hallaron que esta profesora no se enfocó en la cultura franco-anglófona de Montreal y la clase se centró más en ejercicios o actividades lingüísticas, aunque cuando el foco era en el significado más que en la forma se originaron más ocasiones de intercambios culturales, siendo la adaptación y la información cultural los temas más prominentes. Por lo tanto, aunque no se constató que se evidenciara realmente la interculturalidad, esta profesora sí se interesó por las opiniones y experiencias de sus alumnos, al igual que los grupos chilenos del estudio de Menard-Warwick (2009). Dytynyshyn y Collins (2012) destacan también la importancia de que la profesora agrupara a los alumnos por intereses comunes, edades similares, etc., ya que, afirman, la interculturalidad puede crecer cuando existe en el grupo una relación de compañerismo y confianza. Señalan como una limitación de su estudio el que solo se transcribieron las conversaciones de la clase con la profesora y no las conversaciones entre estudiantes trabajando en grupos pequeños, en las que - piensan- se pudieron dar otros ejemplos de faultlines discursivos.

Muy diferentes resultados obtuvieron Magos y Simopoulos (2009) en una investigación de SL sobre si los profesores de griego que impartían clases a adultos inmigrantes de diferentes etnias en Atenas promovían comunicaciones interculturales en sus clases. Realizaron entrevistas semiestructuradas con 20 profesores y 22 alumnos de diferentes cursos y también observaron sus clases. Constataron que solo cuatro de los 20 profesores usaron las experiencias personales de sus alumnos como un recurso cultural en la clase, no solo para crear una atmósfera amigable en la clase si no como un medio hacia la cooperación efectiva. Por ejemplo, en la discusión de un texto sobre una sociedad multicultural (Londres) se incorporó la información que dieron los alumnos sobre su propio país y sobre esas mismas experiencias en Grecia (p. 258). En contraste con esta postura, en la mayoría, de una manera u otra, quedó patente que los profesores veían la cultura griega como una cultura superior a la de sus alumnos, solo siendo más receptivos a perspectivas culturales de países más afines.

"La percepción de los profesores sobre el significado de "cercanía" o "distancia" no escapaba a prejuicios sobre los "otros", incluyendo también estereotipos y comentarios evaluativos de los otros" ["Teachers perceptions of the meaning of cultural "closeness" or "distance" were not free from stereotypes about the "others", while there were also stereotypes and evaluative comments about the "others"] (p. 261). Estas actitudes fueron percibidas por los alumnos que reaccionaron abandonando la clase. Los autores concluyen que estos profesores realmente carecían de entrenamiento en valores y perspectivas interculturales. Por lo tanto, es interesante que sea precisamente esta ausencia de desarrollo de la competencia transcultural en el aula la que haga fracasar una clase de aprendizaje de lengua.

A la luz de los estudios anteriores y dada la importancia en los currículos de lenguas del desarrollo de la competencia transcultural, en este estudio nos proponemos analizar el discurso de clase de diferentes cursos en el extranjero con objeto de discernir si realmente estas discusiones ayudan a problematizar las representaciones culturales de diferentes aspectos de las comunidades en las que los alumnos viven durante unos meses. Respecto a estudios anteriores, el presente estudio se realizó en clases pequeñas (como propusieron Dytynyshyn y Collins, 2012), en una situación de contexto de inmersión cultural con alumnos de niveles avanzado e intermedio, similar al grupo de inmigrantes en California de Menard-Warwick (2009), pero de origen universitario y asistiendo a una clase de claro contenido cultural y conversacional sin unas metas lingüísticas pautadas. Así pues, la pregunta de investigación que nos planteamos es la siguiente: 
¿El discurso de aula de los cursos de contenido cultural en un programa de inmersión contiene rasgos que ayuden al desarrollo de la competencia transcultural?

Investigamos, por lo tanto, si las clases de contenido cultural de los programas de inmersión proporcionan ocasiones para desarrollar la competencia transcultural (Modern Language Association, 2007) adoptando el constructo de faultlines discursivos o diferencias y malentendidos culturales, propuesto por Menard-Warwick (2009). A priori pensamos que el contexto de estudio en el extranjero debería hacer a los alumnos más conscientes de estas diferencias, desencadenadas no solo por el material utilizado en las clases sino también por las observaciones que hacen fueran de clase, al igual que los alumnos inmigrantes de ESL en California del estudio de Menard-Warwick.

\section{El estudio}

\subsection{Participantes}

Este estudio se llevó a cabo con tres cursos diferentes de español como segunda lengua de contenido cultural y conversacional en un programa de estudios en el extranjero en Madrid en el año académico 2011-2012. Se grabaron en vídeo tres clases de tres cursos con estudiantes de nivel intermedio y avanzado. Consideramos en este estudio alumnos de nivel intermedio a aquellos que estaban cursando su tercer y cuarto semestre de español. Los alumnos avanzados tenían todos 6 o más semestres de experiencia con el español. Las tres clases filmadas eran parte de los siguientes cursos, de hora y veinte minutos de duración: "España Hoy" con diez alumnos, "Culturas Hispánicas en los EE.UU. y España”, con siete alumnos y el curso de nivel avanzado "Blogging España", con siete alumnos, dos de estos cursos los impartía el mismo profesor.

Se transcribió el discurso generado, centrándonos en la interacción oral del profesor y de los alumnos. En estas clases, los alumnos se sentaban en semicírculo y el profesor estaba sentado al frente o moviéndose entre los alumnos cuando llevaban a cabo actividades en tríos o en parejas. En estas aulas se respiraba en todo momento una atmósfera relajada y amigable, tanto entre alumnos como entre alumnos y profesor/a. La mayoría del tiempo el español fue la lengua usada en exclusiva por los alumnos y profesores. Las clases fueron grabadas con una cámara portátil instalada en un punto fijo del aula y dirigida hacia los alumnos. La asignatura de "España Hoy” se grabó durante el semestre de otoño de 2011 y las otras dos, la primavera siguiente.

\subsection{Descripción de los cursos}

Los tres cursos se enfocaban en discusión de temas culturales relacionados con la cultura española o hispánica sin tener un objetivo lingüístico convencional. Como Pica (2002) señala, este tipo de cursos culturales pueden facilitar ocasiones para negociaciones de significado y otro tipo de intervenciones del profesor enfocadas en la forma propiciadas por la propia naturaleza conversacional de la clase.

"España Hoy" trata temas de literatura, cine y problemáticas políticas y sociales de España. Cuando se filmó la clase los alumnos estuvieron discutiendo en parejas un par de artículos de prensa, uno relacionado con el impacto de la crisis económica en familias de pocos 
recursos y otro sobre la prohibición de llevar el hijab en las escuelas españolas. Después, los alumnos hablaron entre ellos y con el profesor sobre estos dos temas.

En "Culturas Hispánicas en los EE.UU. y España” se examinan temas relacionados con la presencia de la cultura hispánica en Estados Unidos a través de diferentes materiales como cuentos cortos, noticias, prensa, vídeos, películas, etc. Además, los alumnos también investigan los flujos de inmigración latina en España y su contribución política y cultural en los cambios de España de los últimos años. Durante la clase filmada los alumnos estuvieron involucrados en discusión de clase tanto en pequeños grupos como con toda la clase. Cada alumno tenía que presentar a sus compañeros una noticia relacionada con hispanos aparecida en la prensa reciente. También prepararon una supuesta entrevista entre un funcionario de inmigración y un emigrante sospechoso de contraer matrimonio con el propósito de obtener la licencia de residencia. Una tercera actividad que se desarrolló durante esta sesión de clase fue la presentación por dos alumnos de dos personajes hispanos famosos: Shakira y Messi.

"Blogging España" se centra en el estudio de diferentes géneros periodísticos para desarrollar a través de su práctica la habilidad escrita de los alumnos. Para aprender sobre la representación de la cultura española en los medio de comunicación, los alumnos examinan diferentes artículos de prensa, podcasts, así como programas de radio y televisión que tratan de temas como la inmigración, las diferencias regionales, la crisis económica, etc. Asimismo, los alumnos investigan la representación de los asuntos políticos y culturales en la prensa estadounidense. Todos estos temas se documentan en un blog personal escrito por los alumnos. La clase grabada consistió en presentaciones individuales de diferentes noticias importantes encontradas en la prensa del día, seguidas de discusión del grupo sobre las mismas. Después examinaron juntos una noticia de un telediario y discutieron el contenido y el nuevo vocabulario aprendido. Esta actividad fue liderada directamente por el profesor aunque los alumnos también hablaron entre ellos.

\section{Resultados relacionados con la competencia transcultural en el aula}

Realizamos un análisis temático (Braun y Clarke, 2006) de cada una de las grabaciones de clase. En este análisis temático decidimos codificar aquellos momentos que mostraban cierto desacuerdo entre el profesor y los alumnos, de forma que se pudiera ver algún grado de tensión o sirviera de índice de un faultline discursivo. Las codificaciones fueron evaluadas y discutidas por las dos investigadoras, de forma que la interpretación fuera compartida hasta llegar a un acuerdo en el criterio. A continuación se transcribió el discurso de aula palabra por palabra, siguiendo las convenciones de las transcripciones de la Tabla 1, adaptadas de MenardWarwick (2009, p. 35).

Tabla 1. Convenciones de la transcripción (adaptadas de Menard-Warwick, 2009)

\begin{tabular}{|l|l|}
\hline$[\ldots]$ & Texto omitido \\
{$[$ texto $]$} & Paráfrasis o nota de las autoras \\
$\ldots$ & Entonación alargada \\
Bastardilla & Énfasis \\
() & Dudas de la transcripción \\
$(())$ & Comentario sobre la voz o rasgos paralingüísticos (por ejemplo, risa, gestos) \\
$()$. & Pausa \\
$(.)$. & Pausa larga \\
\hline
\end{tabular}


En "España Hoy" ocurrieron dos instancias de "diferencias culturales o malos entendidos" (Menard-Warwick, 2009). Lo interesante de estos dos casos es que ambos fueron sobre temas relacionados con la cultura local que los alumnos trajeron a la clase como fruto de sus observaciones fuera de esta. En el siguiente fragmento vemos cómo el estudiante se esfuerza no solo en entender el significado de la palabra "hostia", palabra utilizada frecuentemente en jerga coloquial juvenil, sino también en captar su fuerza ilocutiva, como una blasfemia o como un halago.

\section{Fragmento 1}

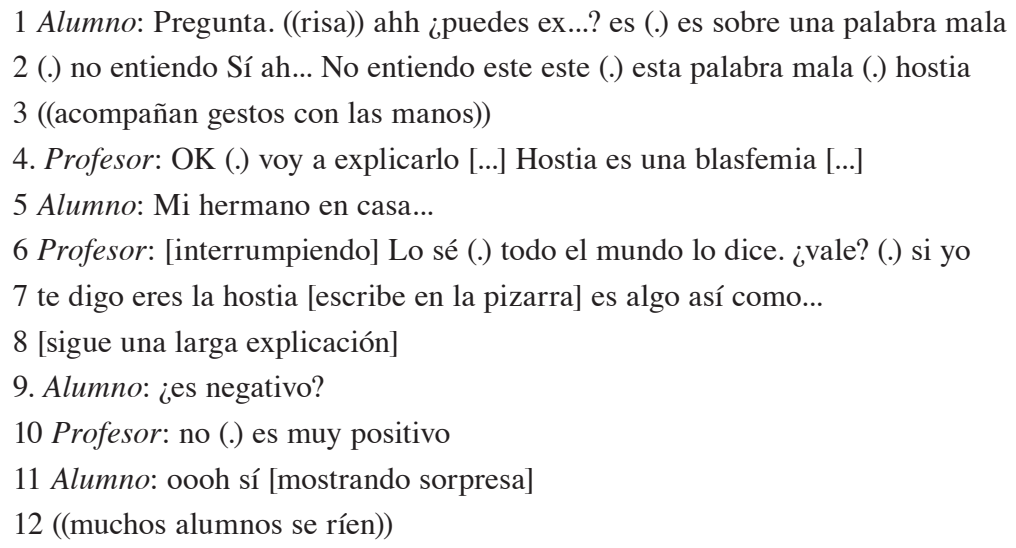

Este es un tipo de discurso que sería difícil que se diera fuera de un discurso de contexto de inmersión. Es decir, el estudiante oye frecuentemente una palabra de la que conoce su significado referencial pero no pragmático. Este tipo de preguntas en la clase apunta a que los estudiantes no solo quieren aprender la lengua sino convertirse en "usuarios" de la misma y toman conciencia de que están proyectando una imagen de sí mismos en base a las opciones lingüísticas que realizan. Si deciden usar la palabra "hostia", están siendo conscientes de que intentan construir una imagen de hablante joven y "enrollado" (cool, para un estadounidense) pero todavía necesitan instrucción por parte del profesor sobre la fuerza real de la palabra. La traducción no ayuda: conocen el significado referencial religioso de la palabra y no se trata de ofender a nadie. Consciente de que es una "mala palabra" (línea 2) no sabe, sin embargo, evaluar el grado de fuerza que tiene ya que se la oye decir a su hermano español con demasiada frecuencia para que pueda ser tan mala (línea 5). Una vez comprendido el significado de la palabra, el alumno se plantea si realmente sabrá usarla en el contexto adecuado.

Este fragmento muestra que los alumnos buscan modelos de lengua que se alejen de los modelos estandarizados de los libros de texto y en el discurso de la clase de idiomas. Este encuentro con normas más locales, menos estandarizadas, de la lengua puede provocar confusión en un primer momento pero también puede ayudar al alumno a desarrollar una identidad a través del lenguaje. Como algunos estudios proponen (Block, 2007; Benson, Barkhuizen, Bodycott y Brown, 2013), las decisiones lingüísticas están en el corazón de esa identidad.

La segunda instancia de negociación de diferencias culturales se desencadenó en esta clase de "España Hoy". El origen de este faultline discursivo fue una protesta de padres divorciados en defensa de su derecho de custodia de sus hijos, que tuvo lugar en la Puerta del Sol en Madrid y que un alumno presenció. Como en el ejemplo anterior, el estudiante recurre 
a la clase para aclarar las dudas que esta experiencia ha suscitado en él. En concreto, en este caso, el alumno duda de si entendió bien los hechos: él sí entendió las pancartas de la protesta pero las diferencias entre estos mensajes y los de su propio estado eran tan grandes que le hizo dudar de que realmente estuviera entendiendo la situación (línea 3). En la experiencia propia de este alumno no era tan evidente que las mujeres recibieran sistemáticamente la custodia de los hijos, salvo en raras excepciones. Reproducimos el fragmento a continuación.

\section{Fragmento 2}

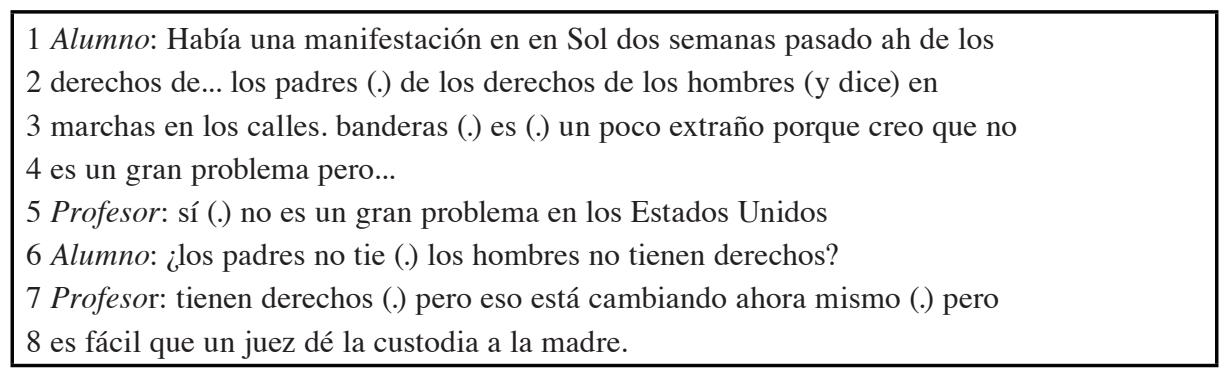

Aunque las lecturas de clase trataron el tema de quién se queda con la custodia de los hijos de divorciados en España, este alumno no reconsideró críticamente este tema hasta que vio esta manifestación en la calle. El estudiante no comprendió bien la escena de la calle precisamente porque la ley de custodia paterna es diferente en estos dos países. En este caso, como ocurrió con los alumnos de Chile (Menard-Warwick, 2008; 2009) y Montreal (Dytynyshyn y Collins, 2012), es la comparación con la propia cultura lo que propicia el faultline, ya que desde la interpretación de la propia cultura no tenía sentido esta manifestación. En este momento la profesora se dio cuenta de que el alumno ponía en duda su comprensión de los hechos precisamente por el contraste cultural que estos le suponían. Llama entonces la atención a la clase sobre el carácter machista que esta ley pueda tener y por eso está cambiándose (línea 8), en un intento de que comprendan que la España de hoy es ya un estado más moderno e igualitario que el retrato de España que hace esta ley.

Vemos entonces, al igual que en los estudios anteriormente mencionados, y a diferencia de los resultados de Magos y Simopoulos (2009), que los profesores del presente estudio sí promueven espacios discursivos sobre los textos, vídeos, etc. sobre la cultura meta para la comprensión del "otro" teniendo en cuenta la perspectiva del aprendiz. Un estudiante del estudio de Magos y Simopoulos (2009, p. 260) comentaba que habría estado bien que cada alumno escribiera sobre un tema y después comparar Grecia con los países de origen. Una diferencia importante, al menos respecto a los profesores de nuestro estudio, es que los profesores griegos no parecen tener una experiencia internacional, mientras que los dos profesores de Madrid se formaron y vivieron durante varios años en EE.UU. Por lo tanto, sin tener probablemente una formación específica en la competencia intercultural sí parecen tener una concienciación cultural suficiente como para integrar y resolver este tipo de faultlines discursivos en sus aulas.

Por último, reproducimos un tercer fragmento del discurso de clase que se produjo en "Blogging España", en el que un alumno trajo a colación otra observación sobre el hecho de que no hubiera camisetas falsas, es decir, ilegales o pirateadas del equipo de fútbol del Real Madrid en mercadillos o tiendas. Los alumnos comentaron cómo esto les chocaba, ya que en otros países se podía conseguir camisetas falsas de cualquier equipo europeo o mundial importante, como en Italia o en Argentina. Es interesante hacer notar que en nuestro grupo de 
alumnos, aunque comparte EE.UU. como lugar de residencia, no son todos estadounidenses y además muchos de ellos vienen ya con otras experiencias internacionales. En nuestro caso, esta acumulación de vivencias se proyecta en la clase, como ocurría en Dytynyshyn y Collins (2012), tal y como se puede ver en el siguiente fragmento.

\section{Fragmento 3}

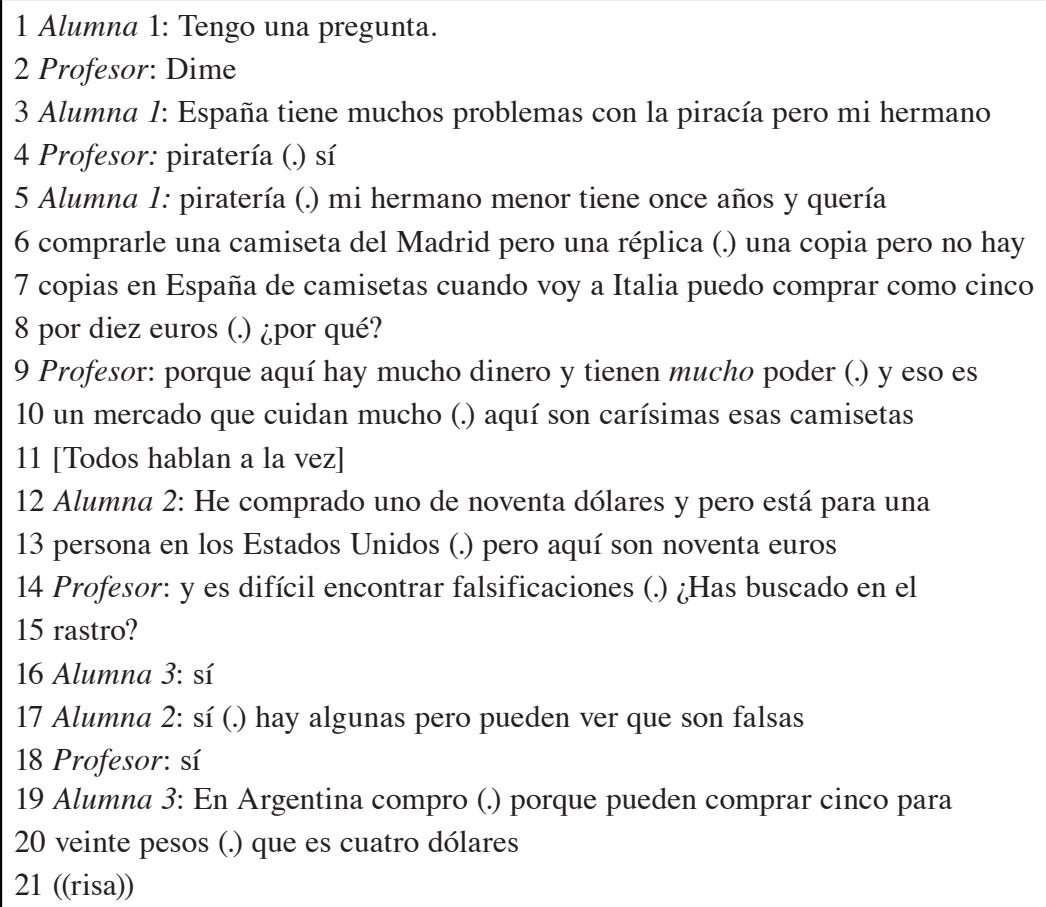

En resumen, los tres fragmentos muestran que los límites entre el contenido de clase y las experiencias fuera de clase se fusionan, produciendo un discurso de aula rico y complejo desde diferentes perspectivas y ángulos, añadiendo profundidad a la información que ofrecen los libros de texto, las lecturas, los vídeos y demás material didáctico.

Las observaciones casuales de los alumnos se vinculan a los temas tratados en clase, agregando esta experiencia al contenido de clase, que de lo contrario sería inerte. El fragmento 1 no está relacionado con el plan de estudios oficial de la clase pero la profesora parece considerar que la clase de cultura es el marco apropiado donde puede discutirse el valor pragmático de una expresión en particular. El estudiante puede estar pensando en incorporar esta palabra a su repertorio léxico, pero él es consciente de su dificultad de uso en una acepción que no es la puramente denotativa. Esta opción léxica con una acepción más coloquial podría afectar a la identidad que este alumno proyecta al oyente.

Por lo tanto, el análisis del discurso de la clase arroja evidencia de que los aprendices no solo participan en una dialéctica derivada de las lecturas, vídeos y otros materiales de clase, sino que tienen que confrontar esa información con la experiencia propia fuera de la clase. Y esto solo puede pasar en programas de inmersión en el extranjero, donde los alumnos están rodeados de estímulos, que, si se discuten debidamente en clase, esto puede agregar una perspectiva más matizada de la cultura meta. 
Una de las facetas de la competencia transcultural es ir más allá de hechos culturales superficiales, contrastando aspectos culturales desde una aproximación respetuosa y sin emitir juicios. Los alumnos de este estudio, con la ayuda y guía de sus profesores, manifestaron sus faultlines discursivos, los cuales actuaron como detonantes para un análisis más profundo de temas generales, tales como los usos reales de palabras malsonantes, las diferencias de género o la piratería de productos en España. Los programas de inmersión son escenarios en los que nuestros alumnos se enfrentan muy a menudo a situaciones de malentendidos culturales, ya que los aprendices están expuestos a múltiples fuentes de información y hay muchas oportunidades de encontrar aspectos no estandarizados de la cultura meta. Incluso, aunque la información sea la misma del texto (como ocurre en el caso del fragmento 2), la relevancia de estos encuentros culturales hacen que los alumnos reflexionen sobre los mismos.

Finalmente, este estudio aporta la ventaja de haber grabado tres clases con un grupo reducido de alumnos que se conocen bien y entre los que existe camaradería y confianza, como señalaba en su estudio Dytynyshyn y Collins (2012) como condiciones favorecedoras para situaciones de intercambio cultural. Sin embargo, solo se pudo grabar un número reducido de horas, lo que supone una clara limitación a las generalizaciones que se puedan hacer respecto a la frecuencia e importancia de este tipo de faultlines e intercambios culturales en nuestras clases y, por extensión, en otros programas. Aunque el interés nuestro fue centrarnos exclusivamente en el discurso de la clase, y por restricciones de tiempo no se tomaron más datos, no deja de ser también una limitación del presente estudio el no contar con entrevistas directas a los profesores y los alumnos de las clases filmadas.

\section{Conclusiones}

El estudio aquí descrito se llevó a cabo en clases pequeñas en una situación de contexto de inmersión con alumnos universitarios de niveles avanzado e intermedio. Se grabaron tres clases enfocadas en temas culturales y conversacionales, sin metas linguiísticas específicas, con el objetivo de investigar si este tipo de cursos proporciona ocasiones que favorezcan al desarrollo de la competencia transcultural.

Parece evidente que en las tres clases observadas se dieron discusiones espontáneas en las que los alumnos hacen referencia a sus vivencias y observaciones fuera del aula, estableciendo un puente entre los contenidos de las clases y sus propias experiencias como extranjeros en Madrid. Sus expectativas no están en consonancia con lo vivido u observado, de ahí que surja el faultline discursivo y el malentendido cultural. La labor del profesor, como conocedor experto de la cultura en la que los alumnos viven, es crucial para que el malentendido llegue a convertirse en una ventana hacia la competencia transcultural (Vande Berg, 2009).

Quizá los ejemplos que se han discutido no sean suficientes para que realmente la competencia transcultural sufra una modificación trascendental, pero lo que nos queda claro es que al realizar estas interacciones en su aula con el profesor, los alumnos están en vías de cuestionarse la realidad circundante, por lo que nos muestran que tienen sus ojos y sus oídos abiertos a las prácticas de la comunidad en la que viven. Y son, efectivamente, estas observaciones las que les hacen dudar y pensar que no están dotando de significado "correcto" a las prácticas, siempre mediadas por su propia cultura estadounidense. Sin embargo, consideramos que es la duda y la pregunta lo que les lleva a una problematización de lo que viven, en lugar de quedarse con una visión cómoda, simplificada y coherente que les deje tranquilos y no les lleve a indagar más sobre las prácticas de esa comunidad que empiezan a conocer. 
En el futuro, y para triangular los resultados que aquí se han ofrecido, se debería visionar con los alumnos los fragmentos grabados en video que hemos analizado para poder contrastar nuestra interpretación con la de los propios alumnos. Con la metodología llamada "think aloud protocol" (pensar en voz alta) se puede elicitar qué estaban pensando los alumnos en el momento en el que hicieron esa intervención para ver si nuestras interpretaciones del discurso generado en el aula coinciden con la de los alumnos. Asimismo, se debería preguntar al profesor correspondiente por el significado de estos fragmentos y si considera que otro tipo de intervención pedagógica podría haber sido más efectiva en el desarrollo de la competencia transcultural de los alumnos.

Las implicaciones pedagógicas que se derivan de este estudio están claramente relacionadas con la importancia del diálogo en las clases para aumentar la curiosidad y la flexibilidad de los puntos de vista adoptados por los alumnos. Es importante, por lo tanto, que el profesor en situación de inmersión reserve tiempo de clase para elicitar dudas lingüísticas motivadas por inseguridad en el contexto cultural adecuado en el que un determinado ítem lingüístico debe producirse (como en el fragmento 1 relacionado con la palabra "hostia"); motivadas por desconocimiento cultural (como en el caso de la custodia para los padres) o simplemente por el contraste cultural (como en el caso de la disponibilidad de falsificaciones). En cualquier caso, el discurso de aula debe favorecer estas intervenciones no planeadas para que la clase actúe de puente entre las dos culturas y empiece a emerger ese tercer espacio (Kramsch, 1993; 2009) desde donde entender mejor ambas, alejándose así de posturas etnocéntricas. Como nos han señalado varios de los autores mencionados en el presente estudio (Dytynyshyn y Collins, 2013; Menard-Warwick, 2008; Magos y Simopoulos, 2009; entre otros), la formación del profesorado en la competencia intercultural es imprescindible para la enseñanza de las lenguas modernas hoy en día. En concreto, en la formación del profesorado se tiene que tener en cuenta la particularidad de las clases, en las que no solo el profesor, los materiales de clase o los otros alumnos pueden ser fuente de malentendido cultural, sino que, como se ha mostrado, las observaciones y experiencias fuera del aula cobran un protagonismo claro.

\section{Bibliografía}

Bacon, S. M. (2002). Learning the rules: Language development and cultural adjustment during study abroad. Foreign Language Annals. 35 (6), 637-646.

Benson, P., Barkhuizen, G., Bodycott, P., y Brown, J. (2013). Second language identity in narratives of study abroad. Nueva York: Palgrave Macmillan.

Block, D. (2007). The rise of identity in SLA research, post Firth and Wagner (1997). The Modern Language Journal. 91 (1), 863-876.

Braun, V., y Clarke, V. (2006). Using thematic analysis in psychology. Qualitative research in psychology. 3 (2), 77-101.

Byram, M. (1997). Teaching and assessing intercultural communicative competence. Clevedon: Multilingual Matters.

Byram, M., Nichols, A., y Stevens, D. (2001). Developing the intercultural dimension in practice. Clevedon: Multilingual Matters. 
Doerr, N. M. (2012). Study abroad as 'adventure': Globalist construction of host-home hierarchy and governed adventurer subjects. Critical Discourse Studies. 9 (3), 257-268.

Doerr, N. M. (2013). Do 'global citizens' need the parochial cultural other? Discourse of immersion in study abroad and learning-by-doing. Compare. 43 (2), 224-243.

Dytynyshyn, N., y Collins, L. (2012). Culture and Interculturality in the Adult ESL Context in Urban Quebec: A Case Study. TESL Canada Journal. 30 (1), 45-68.

Goldoni, F. (2013). Students' immersion experiences in study abroad. Foreign Language Annals. 46 (3), 359-376.

Gore, J. E. (2005). Dominant beliefs and alternative voices: Discourse, belief, and gender in American study abroad. Nueva York: Routledge.

Howard, M. (2001). The effects of study abroad on the L2 learner's structural skills: Evidence from advanced learners of French. Eurosla Yearbook. 1, 123-141.

Howard, M. (2005). On the role of context in the development of learner language: Insights from study abroad research. International Review of Applied Linguistics. 148, 1-20.

Jackson, J. (2008). Language, Identity and Study Abroad. Sociocultural Perspectives. Londres: Equinox.

Kramsch, C. (1993). Context and culture in language teaching. Oxford: Oxford University Press.

Kramsch, C. (2009). Third Culture and Language Education. Por L. Weis y V. Cook (Eds.), Contemporary Applied Linguistics. (Vol. 1). (233-254). Londres: Continuum.

Magos, K., y Simopoulos, G. (2009). 'Do you know Naomi?’: Researching the intercultural competence of teachers who teach Greek as a second language in immigrant classes. Intercultural Education. 20 (3), 255-265.

Martínez-Arbelaiz, A., y Pereira, I. (2008). La adquisición del pretérito imperfecto en situación de inmersión. Spanish in context. 5 (2), 161-181.

Meara, P. (1994). The year abroad and its effects. Language Learning Journal. 10, 32-38.

Menard-Warwick, J. (2009). Co-constructing Representations of Culture in ESL and EFL Classrooms: Discursive Faultlines in Chile and California. The Modern Language Journal. 93 (1), 30-45.

Menard-Warwick, J. (2008). The cultural and intercultural identities of transnational English teachers: Two case studies from the Americas. TESOL quarterly. 42 (4), 617-640.

Merino, E., y Avello, P. (2014). Contrasting intercultural awareness at home and abroad. Por C. Pérez-Vidal (Ed.). Language acquisition in study abroad and formal instruction contexts. (Vol. 13). (283-309). Amsterdam: John Benjamins Publishing Company.

Milton, J., y Meara, P. (1995). How periods abroad affect vocabulary growth in a foreign language. ITL Institut voor Togepaste Linguistik. 107-108, 17-34.

Modern Language Association (2007). Foreign Language and Higher Education. New Structures for a Changed World. http://www.mla.org/flreport [Consulta 10 de enero de 2014].

Pérez-Vidal, C. (Ed.). (2014). Language acquisition in study abroad and formal instruction contexts. (Vol. 13). Amsterdam: John Benjamins Publishing Company. 
Pérez-Vidal, C., y Juan-Garau, M. (2009). The effect of Study Abroad (SA) on written performance. Eurosla yearbook. 9 (1), 269-295.

Pica, T. (2002). Subject-Matter Content: How does it assist the Interactional and Linguistic Needs of Classroom Language Learners? The Modern Language Journal. 86 (1), 1-19.

Segalowitz, N., y Freed, B. A. (2004). Context, contact, and cognition in oral fluency acquisition. Studies in Second Language Acquisition. 26 (2), 173-199.

Takkula, H., Kangaslahti, J., y Banks, J. (2008). Teaching transcultural competence: From language learning to experiential education. Policy \& Practice-A Development Education Review. 7, 88-95.

Vande Berg, M. (2009). Intervening in student learning abroad: A research-based inquiry. Intercultural Education. 20 (1), S15-S27. 